\title{
Análisis de desgaste para el fresado de muestras de aluminio AA 7075-T6
}

\author{
Augusto Coque, Byron Cortez \\ Centro de Posgrados/Ingeniería/Mecánica Universidad de las Fuerzas Armadas - ESPE, Sangolquí, Ecuador \\ acoque@ups.edu.ec, bhcortez@espe.edu.ec
}

\begin{abstract}
Resumen-La presente investigación describe la influencia de los parámetros de mecanizado en el desgaste de las herramientas y la calidad superficial. Los factores que se consideran para este análisis son: velocidad de corte (Vc), Avance por diente (fz), Profundidad de corte $\left(a_{p}\right)$ y la Trayectoria de la herramienta (T), para lo cual, en un centro de mecanizado EMCO Concept Mill 260 y en una muestra de Aluminio A7075-T6 se realizó una operación de fresado plano. Para el modelo experimental se utilizó el método estadístico Taguchi con un arreglo ortogonal L27. Estableciendo parámetros de mecanizado que ayuden a obtener desgastes mínimos en las herramientas sin influir en los acabados superficiales de las muestras manufacturadas. Acorde a esta investigación el factor (fz) es estadísticamente significante para la rugosidad superficial y los factores (fz) y $\left(a_{p}\right)$ para el desgaste por filo de aportación, estos modelos dan como resultado una rugosidad superficial $0.061 \mu \mathrm{m}$ y una cantidad de $0,004 \mathrm{~mm}^{3}$ de aluminio adheridos a la herramienta. Después de utilizar los parámetros de mecanizado óptimos se obtuvo una mejora de $4,9 \%$ para la rugosidad y una reducción del 7,5\% en el desgaste por filo de aportación.
\end{abstract}

Palabras Claves-Fresado plano, Parámetros de mecanizado, Desgaste por filo de aportación, Rugosidad superficial.

Abstract-Due to its excellent mechanical properties, machinability and relatively low cost compared with other metals, aluminum alloys are highly utilized in the automotive industry, marine, aviation and in plastic injection dies. However, there is limited information related to the machining parameters of aluminum and its alloys, especially in Ecuador. This research describes the influence of machining parameters into the tools-worn out and surface quality. The factors that are considered for this analysis are: cutting speed (Cs), feed per tooth (ft), depth of cut (D.O.C), and tool path (Tp). A machining center EMCO Concept Mill 260 was used as well as Aluminum A7075-T6 specimens. Taguchi methods and an orthogonal array L27 were utilized. The analysis yielded that factor (ft) is statistically significant when evaluating the surface roughness. Factors (ft) and (D.O.C) are significant for the worn out of the blade. It was measured 0.061um for the surface roughness and $0.004 \mathrm{~mm} 3$ adhered to the cutting tool. After optimizing the parameters, an improvement of $4.9 \%$ was evidenced for the surface roughness and a reduction of $7.5 \%$ in the worn out of the tools.

Keywords - Flat milling, Machining parameters, Built Up Edge, Surface roughness.

\section{INTRODUCCIÓN}

En la actualidad existen una gran cantidad de materiales que presentan muy buenas propiedades para determinadas aplicaciones, el aluminio es un material que desde su descubrimiento ha aportado en un sin número de campos de aplicación, este metal estructural es el más abundante en la corteza terrestre con un valor aproximado del 7,5\%.
La aleación de aluminio A7075-T6 (Prodax) es un material que tiene excelentes propiedades mecánicas como por ejemplo una buena resistencia a la fatiga, además es ligero y de fácil mecanizado frente a otros metales, debido a su costo relativamente accesible, su uso es habitual en aplicaciones donde las características técnicas de aleaciones más baratas no son admisibles.

En el mecanizado influyen diversas variables las cuales se pueden dividir en independientes y dependientes, minimizar el desgaste de las herramientas y mantener la calidad superficial de los elementos fabricados es uno de los paradigmas del mecanizado, al variar los factores independientes tales como los parámetros de corte en un proceso de fresado plano se buscará encontrar cuales son los más influyentes en el desgaste de las herramientas, sin afectar el acabado superficial de las piezas mecanizadas en un Centro de Mecanizado CNC.

Se realizará un diseño experimental basado en la Metodología Taguchi, el cual es utilizado extensamente en la industria y estudios científicos, así se determinará la optimización del proceso de fresado plano encontrando los parámetros de corte que nos ayuden a minimizar el desgate de las herramientas por filo de aportación (BUE del inglés Built Up Edge) y la calidad superficial de una pieza mecanizada.

\section{MÉTODO}

Para encontrar y optimizar los parámetros de mecanizado más influyentes en el desgate de las herramientas por filo de aportación y la rugosidad superficial, al mecanizar la aleación de aluminio A7075-T6 (Prodax), en un Centro de Mecanizado EMCO Concept Mill 260, realizando un proceso de fresado plano, utilizando una herramienta de diámetro $50 \mathrm{~mm}$ con insertos de metal duro, mediante ensayos experimentales.

Se planteará el diseño de la metodología experimental, utilizando el método Taguchi y la metodología ANOVA, los cuales nos ayudarán a obtener los parámetros de mecanizado más influyentes en el desgaste de las herramientas por filo de aportación (BUE) y el acabado superficial, para luego encontrar los parámetros de mecanizado óptimos en el fresado plano.

Se realizará microfotografías de los tipos de desgaste encontrados evaluando los resultados para obtener los parámetros de mecanizado óptimos que disminuyan el BUE de las herramientas y mejoren la calidad superficial.

Mediante el fresado plano a través de la variación de parámetros de corte establecidos en la Tabla I para el material, utilizando una herramienta de carburo no recubierto (metal duro), realizar un análisis detenido para controlar todos los factores de mecanizado influyentes sería 
demasiado extenso, ya que sin duda son muchos más que los antes mencionados por lo cual se ha optado por obtener datos viables, concisos y útiles, definiendo una forma rectangular de la probeta con las siguientes dimensiones 100x75x30 como se muestra en la Figura 1, el diámetro de la herramienta (diámetro $50 \mathrm{~mm}$ ), y la no utilización de refrigerante en el centro de mecanizado, al realizar el planeado sobre la superficie se seleccionará tres estrategias de corte.

TABLA I FRESADO PLANO Y DE ESCUADRADO

\begin{tabular}{|c|c|c|c|c|}
\hline & $\begin{array}{c}\text { Fresado } \\
\text { grueso con } \\
\text { metal duro }\end{array}$ & $\begin{array}{c}\text { Fresado } \\
\text { fino con } \\
\text { metal duro }\end{array}$ & $\begin{array}{c}\text { Fresado } \\
\text { fino con } \\
\text { PCD }^{1)}\end{array}$ & $\begin{array}{c}\text { Fresado } \\
\text { con acero } \\
\text { rápido }\end{array}$ \\
\hline $\begin{array}{l}\text { Velocidad } \\
\text { de corte } \\
\text { m/min } \\
\text { a.p.m }\end{array}$ & $\begin{array}{c}600-1000 \\
1980-3300\end{array}$ & $\begin{array}{l}1000-3000 \\
3300-9900\end{array}$ & $\begin{array}{c}800-4000 \\
2650-13200\end{array}$ & $\begin{array}{c}250-400 \\
825-1320\end{array}$ \\
\hline $\begin{array}{l}\text { Avance }\left(f_{z}\right) \\
\text { mm/diente } \\
\text { Pulg/diente }\end{array}$ & $\begin{array}{c}0,2-0,6 \\
0,008-0,024\end{array}$ & $\begin{array}{c}0,1-0,2 \\
0,004-0,008\end{array}$ & $\begin{array}{c}0,05-0,2 \\
0,002-0,008\end{array}$ & $\begin{array}{c}-0,4 \\
-0,016\end{array}$ \\
\hline $\begin{array}{c}\text { Profundidad } \\
\text { de corte }\left(a_{p}\right) \\
\text { Mm } \\
\text { Pulgadas }\end{array}$ & $\begin{array}{c}2-8 \\
0,08-0,32\end{array}$ & $\begin{array}{c}-2 \\
-0,08\end{array}$ & $\begin{array}{c}-2 \\
-0,08\end{array}$ & $\begin{array}{c}-8 \\
-0,32\end{array}$ \\
\hline $\begin{array}{c}\text { Designación } \\
\text { del metal } \\
\text { duro ISO }\end{array}$ & K20 & K10 & - & - \\
\hline
\end{tabular}

La herramienta de corte seleccionada es ZCC-CT fresa FMR04, 50 mm de diámetro y cuatro dientes , [1] en su estudio utilizan solo un inserto para facilitar la detección del desgaste en el mecanizado de Inconel-182 producido por la temperatura, para la elección de la herramienta FMR04 con insertos YD201-SEKN1203AFTN no recubiertos se puede justificar su uso ya que la remoción de material es lo más importante para la mayoría de fabricantes primordialmente en la fabricación de moldes y en el campo aeroespacial en el cual el Aluminio Prodax es muy utilizado [2].

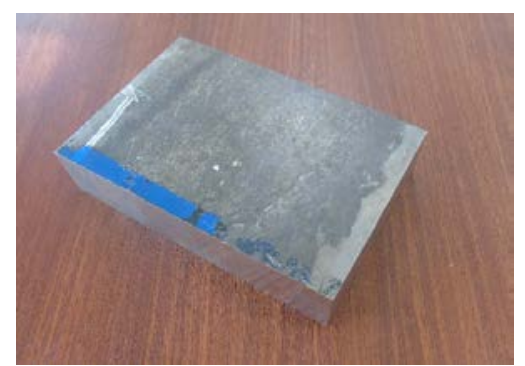

Fig. 1. Probeta aluminio

La Tabla II muestra los valores de mecanizado recomendados para la herramienta de corte FMR04, mientras que en la Tabla III se observa los valores recomendados para los insertos YD201-SEKN1203AFTN.

El Dr. Genichi Taguchi propuso un método estadístico en 1960 el cual es extensamente usado en la industria y estudios científicos, pues desde que este es aplicado se puede determinar fácilmente la optimización de un proceso y no requiere de cálculos matemáticos complejos.

TABLA II

PARÁMETROS DE CORTE PARA LA HERRAMIENTA FMR01

\begin{tabular}{cc} 
Velocidad de corte $\left(\mathrm{V}_{\mathrm{c}}\right)$ & $>600 \mathrm{~m} / \mathrm{min}$ \\
Avance $\left(\mathrm{f}_{\mathrm{z}}\right)$ & $0,3-0,5 \mathrm{~mm} / \mathrm{z}$ \\
Profundidad de corte $\left(\mathrm{a}_{\mathrm{p}}\right)$ & $2-5 \mathrm{~mm}$ \\
\hline
\end{tabular}

TABLA III

PARÁMETROS DE CORTE PARA INSERTO ES ZCC-CT YD201SEKN1203AFTN

$\begin{array}{cc}\text { Velocidad de corte }\left(\mathrm{V}_{\mathrm{c}}\right) & 160-200 \mathrm{~m} / \mathrm{min} \\ \text { Avance }\left(\mathrm{f}_{\mathrm{z}}\right) & 0,1-0,3 \mathrm{~mm} / \mathrm{z} \\ \text { Profundidad de corte }\left(\mathrm{a}_{\mathrm{p}}\right) & 2-5 \mathrm{~mm}\end{array}$

El sistema de diseño de experimentos de Taguchi se deriva de alrededor de 18 arreglos ortogonales estándar. Un arreglo ortogonal es una matriz experimental factorial fraccional que es ortogonal y balanceada [3].

Para el experimento se debe determinar los factores elegidos y sus niveles los cuales se encuentran en la Tabla IV, estos valores han sido recolectados de las Tablas I, II y III. Especificada la velocidad de corte, calculamos el avance en $\mathrm{mm} / \mathrm{min}$ tomando del catálogo de herramientas el valor del avance por diente, con lo cual tenemos los tres niveles para este factor. Los niveles de la trayectoria de la herramienta serán: de $\mathrm{X}$ a $\mathrm{Y}$ con retracción, de $\mathrm{Y}$ a $\mathrm{X}$ con retracción y de $\mathrm{X}$ a $\mathrm{Y}$ sin retracción. Las profundidades están de acuerdo a la relación profundidad/rugosidad, y profundidad axial permitida por el diámetro de la herramienta por lo cual serán 2 mm, 3mm, y 4mm.

TABLA IV

FACTORES SELECCIONADOS Y SUS NIVELES

\begin{tabular}{llcccc}
\hline \hline & \multicolumn{1}{c}{ Factores } & Unidades & $\mathbf{1}$ & $\mathbf{2}$ & $\mathbf{3}$ \\
\hline A & Velocidad de corte & $\mathrm{m} / \mathrm{min}$ & 160 & 450 & 900 \\
B & Avance por diente & $\mathrm{mm} / \mathrm{z}$ & 0,1 & 0,2 & 0,3 \\
C & Profundidad de corte & ---- & 2 & 3 & 4 \\
D & Trayectoria & $\mathrm{mm}$ & $\mathrm{X}-\mathrm{Y}$ & $\mathrm{Y}-\mathrm{X}$ & $\mathrm{X}-\mathrm{Y}$ \\
\hline \hline
\end{tabular}

Se determinará el arreglo ortogonal a utilizar, ya que el método Taguchi propone que el número de grados de libertad del arreglo ortogonal (DF) que se seleccionará, debe ser mayor o igual al total de grados de libertad requerido para el experimento. La ecuación para calcular los grados de libertad del experimento relacionado al número de factores y niveles está dada por [3]:

$$
D F=[(n 1-1) \cdot n f+(n 1-1) \cdot(n 1-1) \cdot n i]
$$

donde $D F$ son los Grados de libertad del experimento, $n 1$ son los números de niveles, $n f$ son los números de factores y ni son los números de interacciones, mediante (1), se obtiene un resultado de los grados de libertad igual a 20 considerando esto se seleccionará el arreglo ortogonal (AO), L27 el cual tiene $(D F=26)$ este arreglo consta de 27 filas y 13 columnas este será el arreglo seleccionado para el experimento de esta manera el diseño experimental consiste en 27 ensayos.

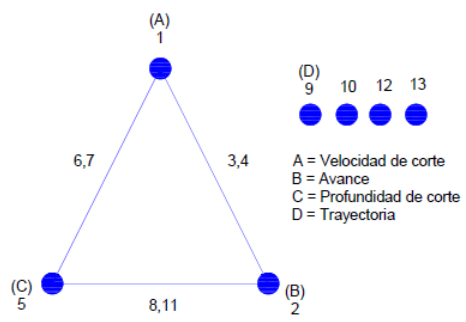

Fig. 2. Aplicación lineal AO L27 
En la Fig. 2 el efecto principal se representa mediante un punto mientras que una interacción se representa mediante una línea y los números las columnas correspondientes del arreglo ortogonal.

Para el desarrollo del experimento se utilizará 27 probetas una para cada ensayo, se realizará el desarrollo del cálculo de los valores necesarios para el mecanizado de los diferentes experimentos, La ecuación de la velocidad de corte está dada por [4]:

$$
V_{C}=\frac{\pi \cdot N \cdot D}{1000}
$$

donde $N$ son las revoluciones por minuto y $D$ el diámetro de la herramienta, se deberá tomar en cuenta el diámetro de corte efectivo que está dado por [4] donde $a_{p}$ es la profundidad de corte y $K r$ el ángulo de mecanizado de la herramienta que para nuestro estudio es $45^{\circ}$.

$$
D e=D+\frac{2 \cdot a_{p}}{\tan k r}
$$

Utilizando la ecuación que está dada por [4]:

$$
V f=f z \cdot z \cdot N
$$

donde $f z$ es el avance por diente y $z$ es el número de filos, obteniéndose el valor de la velocidad de avance de la herramienta.

En la Fig. 3, se muestra una probeta con los parámetros obtenidos anteriormente que nos servirán para el análisis de resultados.
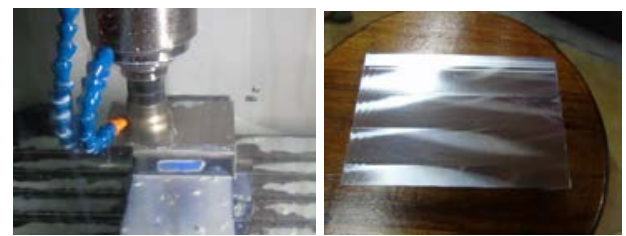

Fig. 3. Mecanizado de Probetas

Se utilizará un microscopio óptico BUEHLER VIEWMET para observar los diferentes tipos de desgaste que se producen en las herramientas, además con un rugosímetro marca MITUTOYO SJ-210 se realizará diferentes ensayos en el laboratorio de metrología de la Universidad Politécnica Salesiana como se observa en la Figura 4, con los datos obtenidos de las observaciones que se realizarán con el microscopio y el rugosímetro, se utilizará el METODO ANOVA.

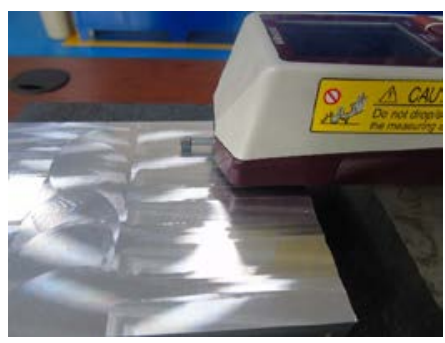

Fig. 4. Medición de la rugosidad superficial

\section{ANÁLISIS DE RESULTADOS}

Al aplicar la Metodología Taguchi con el modelo ortogonal L27 que combina los niveles y factores para el fresado Plano, se obtendrá las medidas de rugosidad de forma perpendicular y paralela al movimiento de la herramienta. Se realizará tres ensayos de medida de rugosidad, obteniéndose de esta manera una rugosidad promedio, uno de los principales problemas en el mecanizado de materiales pastosos como el aluminio, es el material que se adhiere a la herramienta provocando un tipo de desgaste conocido como Filo de aportación (BUE, por sus siglas en inglés). Con en el microscopio BUEHLER VIEWMET se observará el filo de los insertos utilizados en los ensayos realizados, apreciando que existe material de aluminio adherido en el filo de las herramientas. Utilizando el software propio del microscopio BUEHLER VIEWMET se podrá obtener la medida del área y la altura del material adherido en la herramienta como se muestra en la Figura 5.a y b. Los valores obtenidos en el software se encuentran en micras.

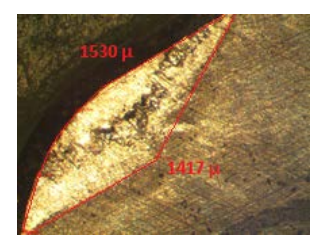

(a)

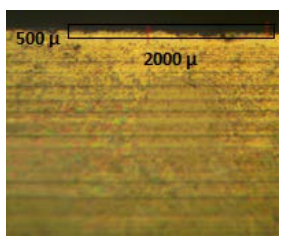

(b)
Fig. 5. Filo de inserto presentando material adherido

La Tabla V muestra el volumen del material adherido a las herramientas utilizadas en los ensayos. Estos valores se obtuvieron al multiplicar el área del material adherido en la parte plana y la altura que se presenta en el filo inclinado como se muestras en la Fig. 5(a) y Fig. 5(b).

Al utilizar el método ANOVA se podrá determinar los factores de mecanizado y sus respectivos niveles óptimos, optimizando la rugosidad medida en sentido paralelo al mecanizado, la rugosidad medida en sentido perpendicular al mecanizado y la cantidad de material adherido a la herramienta.

Al aplicar los parámetros de mecanizado óptimos, las tablas VI, VII y VIII muestran el valor de rugosidad mejorada en sentido paralelo, perpendicular y la cantidad de material adherido a la herramienta respectivamente.

\section{CONCLUSIONES}

Se determinó que $0,004 \mathrm{~mm}^{3}$ es la menor cantidad de material adherido a la herramienta, además los valores de rugosidad más bajos son 0,061 $\mu \mathrm{m}$ para la rugosidad medida en sentido paralelo al mecanizado y 0,068 $\mu \mathrm{m}$ para la rugosidad medida en sentido perpendicular al mecanizado. Se determinó que los parámetros que disminuyen la cantidad de material adherido a la herramienta son: Velocidad de corte $450 \mathrm{~m} / \mathrm{min}$, Avance $0.2 \mathrm{~mm} /$ filo, profundidad de corte $2 \mathrm{~mm}$ y una trayectoria de $\mathrm{x}$ a y sin retracción obteniéndose un volumen de $0.0037 \mathrm{~mm}^{3}$ de material adherido a la herramienta. Se determinó que para la rugosidad medida en sentido paralelo al mecanizado los parámetros que mejoran la rugosidad son: Velocidad de corte $450 \mathrm{~m} / \mathrm{min}$, Avance 0.1 $\mathrm{mm} /$ filo, profundidad de corte $2 \mathrm{~mm}$ y una trayectoria de $\mathrm{x}$ a y sin retracción, obteniéndose una rugosidad mejorada de $0.058 \mu \mathrm{m}$ mejorando en un $4.9 \%$ el acabado superficial, mientras que para la rugosidad medida en sentido 
perpendicular al mecanizado los parámetros que mejoran la rugosidad son: Velocidad de corte $900 \mathrm{~m} / \mathrm{min}$, Avance 0.1 $\mathrm{mm}$ /filo, profundidad de corte $2 \mathrm{~mm}$ y una trayectoria de $\mathrm{x}$ a y sin retracción, obteniéndose una rugosidad mejorada de $0.064 \mu \mathrm{m}$ mejorando en un 5.8\% el acabado superficial.

TABLA V

VOLUMEN DE MATERIAL ADHERIDO EN LA HERRAMIENTA

\begin{tabular}{ccccc}
$\mathbf{N}^{\mathbf{0}}$ Prueba & $\begin{array}{c}\text { Largo } \\
(\mathbf{m m})\end{array}$ & $\begin{array}{c}\text { Ancho } \\
\mathbf{( m m )}\end{array}$ & $\begin{array}{c}\text { Altura } \\
\mathbf{( m m )}\end{array}$ & $\begin{array}{c}\text { Volumen } \\
\mathbf{( m m}^{\mathbf{3}} \mathbf{)}\end{array}$ \\
\hline 1 & 2.60 & 0.010 & 0.500 & 0.013 \\
2 & 3.56 & 0.730 & 0.500 & 1.299 \\
3 & 2.44 & 0.100 & 0.500 & 0.122 \\
4 & 2.26 & 0.150 & 0.500 & 0.169 \\
5 & 2.32 & 0.050 & 0.500 & 0.058 \\
6 & 0.69 & 0.100 & 0.500 & 0.034 \\
7 & 2.27 & 0.070 & 0.500 & 0.079 \\
8 & 2.57 & 0.170 & 0.500 & 0.218 \\
9 & 1.20 & 1.000 & 0.500 & 0.600 \\
10 & 0.57 & 0.020 & 0.500 & 0.006 \\
11 & 1.25 & 0.440 & 0.500 & 0.275 \\
12 & 1.05 & 0.200 & 0.500 & 0.105 \\
13 & 0.58 & 0.015 & 0.500 & 0.004 \\
14 & 2.26 & 0.050 & 0.500 & 0.056 \\
15 & 0.68 & 0.090 & 0.500 & 0.031 \\
16 & 1.05 & 0.015 & 0.500 & 0.008 \\
17 & 1.08 & 0.450 & 0.500 & 0.243 \\
18 & 0.70 & 0.070 & 0.500 & 0.025 \\
19 & 2.10 & 0.150 & 0.500 & 0.157 \\
20 & 1.15 & 0.020 & 0.500 & 0.011 \\
21 & 2.25 & 0.170 & 0.500 & 0.191 \\
22 & 0.70 & 0.090 & 0.500 & 0.031 \\
23 & 1.20 & 0.300 & 0.500 & 0.180 \\
24 & 0.50 & 0.070 & 0.500 & 0.017 \\
25 & 1.13 & 0.170 & 0.500 & 0.096 \\
26 & 0.58 & 0.200 & 0.500 & 0.058 \\
27 & 2.80 & 0.980 & 0.500 & 1.372 \\
\hline \hline
\end{tabular}

TABLA VI

VALOR DE RUGOSIDAD MEJORADA MEDIDA EN SENTIDO PARALELO AL MECANIZADO

\begin{tabular}{|c|c|c|c|c|c|c|c|c|}
\hline $\begin{array}{c}\mathrm{N}^{\circ} \\
\text { Prue } \\
\text { ba }\end{array}$ & $\begin{array}{c}\text { Vc } \\
(\mathbf{m} / \mathbf{m} \\
\text { in) }\end{array}$ & $\begin{array}{c}\mathbf{f z} \\
(\mathbf{m m} / \mathbf{r} \\
\mathbf{e v})\end{array}$ & $\begin{array}{l}a_{p} \\
(m \\
m)\end{array}$ & $\begin{array}{c}\text { Trayect } \\
\text { oria }\end{array}$ & $\begin{array}{c}\text { R1 } \\
(\mu \\
\mathbf{m})\end{array}$ & $\begin{array}{l}\mathrm{R} 2 \\
(\mu \\
\mathrm{m})\end{array}$ & $\begin{array}{l}\text { R3 } \\
(\mu \\
\text { m) }\end{array}$ & $\begin{array}{c}\mathrm{R} \\
\text { prome } \\
\text { dio } \\
(\mu \mathrm{m}) \\
\end{array}$ \\
\hline 1 & 450 & 0,1 & 2 & $\begin{array}{c}\text { De } x \text { a } y \\
\text { CR }\end{array}$ & $\begin{array}{c}0,0 \\
6 \\
\end{array}$ & $\begin{array}{l}0,0 \\
57 \\
\end{array}$ & $\begin{array}{l}0,0 \\
59 \\
\end{array}$ & 0,058 \\
\hline
\end{tabular}

TABLA VII

VALOR DE RUGOSIDAD MEJORADA MEDIDA EN SENTIDO PERPENDICULAR AL MECANIZADO

\begin{tabular}{|c|c|c|c|c|c|c|c|c|}
\hline $\begin{array}{c}N^{o} \\
\text { Pru } \\
\text { eba }\end{array}$ & $\begin{array}{c}\text { Vc } \\
(\mathrm{m} / \\
\mathrm{min})\end{array}$ & $\begin{array}{c}\mathbf{f z} \\
(\mathbf{m m} / \\
\text { rev })\end{array}$ & $\begin{array}{l}a_{p} \\
(m \\
m)\end{array}$ & $\begin{array}{c}\text { Trayec } \\
\text { toria }\end{array}$ & $\begin{array}{c}\text { R1 } \\
(\boldsymbol{\mu} \\
\mathbf{m})\end{array}$ & $\begin{array}{c}\mathrm{R} 2 \\
(\boldsymbol{\mu} \\
\mathrm{m})\end{array}$ & $\begin{array}{l}\text { R3 } \\
(\mu \\
\text { m) }\end{array}$ & $\begin{array}{c}R \\
\text { prom } \\
\text { edio } \\
(\mu \mathrm{m})\end{array}$ \\
\hline 2 & 900 & 0,1 & 2 & $\begin{array}{l}\text { De x a } \\
\text { y CR }\end{array}$ & $\begin{array}{l}0,0 \\
67\end{array}$ & $\begin{array}{l}0,0 \\
63\end{array}$ & $\begin{array}{l}0,0 \\
64\end{array}$ & 0,064 \\
\hline
\end{tabular}

TABLA VIII

VALOR MEJORADO DE CANTIDAD DE MATERIAL ADHERIDO A LA

HERRAMIENTA

\begin{tabular}{|c|c|c|c|c|c|c|c|c|}
\hline $\begin{array}{l}N^{o} \\
\text { Pru } \\
\text { eba }\end{array}$ & $\begin{array}{c}\text { Vc } \\
(\mathbf{m} / \\
\mathrm{min})\end{array}$ & $\begin{array}{c}\mathbf{f z} \\
(\mathrm{mm} / \\
\text { rev })\end{array}$ & $\begin{array}{l}a_{p} \\
(\mathbf{m} \\
\mathbf{m})\end{array}$ & $\begin{array}{c}\text { Trayec } \\
\text { toria }\end{array}$ & $\begin{array}{c}\text { Lar } \\
\text { go } \\
\text { (m } \\
\text { m) }\end{array}$ & $\begin{array}{l}\text { Anc } \\
\text { ho } \\
\text { (m } \\
\text { m) }\end{array}$ & $\begin{array}{l}\text { Alt } \\
\text { ura } \\
(\mathbf{m} \\
\text { m) }\end{array}$ & $\begin{array}{c}\text { Volu } \\
\text { men } \\
\left(\mathrm{mm}^{3}\right. \\
)\end{array}$ \\
\hline 3 & 450 & 0,2 & 2 & $\begin{array}{l}\text { De x a } \\
\text { y CR }\end{array}$ & $\begin{array}{c}0,4 \\
6\end{array}$ & $\begin{array}{c}0,01 \\
3\end{array}$ & $\begin{array}{l}0 \\
48\end{array}$ & $\begin{array}{c}0,003 \\
7\end{array}$ \\
\hline
\end{tabular}

\section{AGRADECIMIENTOS}

Agradezco al Rvdo. Padre Javier Herrán Gómez Rector de la Universidad Politécnica Salesiana por el apoyo financiero y la confianza depositada hacia mi persona en la realización de mis estudios y este proyecto de investigación.
Agradezco al Ing. Byron Cortez MSc. por su guía y su conocimiento pilares fundamentales en el desarrollo de esta investigación.

\section{REFERENCIAS}

[1] W. M. Chengdong Wang, «Milling tool's flank wearpredictionbytemperaturedependentwear,» Tribology International, pp. 140-156, 2016.

[2] M. Danford, «Internacional Metalmecánica,» Octubre 2009. [En línea]. Available: http://www.metalmecanica.com/temas/Material-deherramientas-alternativo-para-desbastar-piezas-de-trabajograndes+7072835. [Último acceso: 0108 2016].

[3] D. C. Montgomery, Diseño y Analisis de experimentos, Mexico DF.: Limusa, 2010.

[4] S. Kalpakjian, Manufactura, Ingeniería y Tecnología, Mexico D.F. Pearson Educación, 2016.

[5] J. C. C. John L. Yang, «A Systematic Approach for Identifying Optimum Surface Roughness Performance in End-Milling Operations,» Industrial Technology, 2001. 